\title{
Incidence of Simian Crease in Normal Nepalese Children
}

\author{
Malla $\mathrm{T}^{1}$, Malla KK², Gauchan $\mathrm{E}^{3}$, Brijesh $\mathrm{S}^{4}$, Sharma $\mathrm{R}^{5}$
}

1,2,3,5 Department of Pediatrics,

${ }^{4}$ Department of Community Medicine

ABSTRACT

Manipal College of Medical Sciences, Phulbari,

Pokhara, Nepal.

Corresponding Author

Dr Tejesh Malla

Department of Pediatrics

Manipal College of Medical Sciences, Phulbari,

Pokhara, Nepal.

Email. tejeshmalla@hotmail.com

Kathmandu Univ Med J 2010;9(32):410-4

\section{Backgroud}

Simian crease is usually associated with some chromosomal anomalies and syndromes but it is also seen in some populations without any chromosomal defects.

\section{Objective}

To see the incidence of simian crease in children without chromosomal anomalies and to detect the Ethnic group variations.

\section{Methods}

A prospective study in children attending the paediatric outpatient department of Manipal Teaching Hospital, Pokhara. $-2,067$ children were screened randomly from the $1^{\text {st }}$ June 2007 to the $31^{\text {st }}$ December 2007. Palm crease and axial triradius angle were detected in every child. Axial Triradius angle was compared between those who have simian crease to those who do not simian crease.Children who were found with simian crease underwent IQ testing. The exclusion criteria were children with Down syndrome, other chromosomal and minor anomalies, plus or any other chronic disease condition.

\section{Results}

2,067 children ( 1,084 boys $\& 983$ girls) were screened. Among them four were cases of Down syndrome so were excluded from the study. Finally total of $2,063(1,082$ boys \& 981 girls) were the study group. There were a total 14 ethnic groups who attended the outpatient department of Manipal Hospital during a seven months period. Among the seven ethnic groups Brahaman, Gurung, Tamang, Lama, Newar, Chettri and Dalit had single palmar crease. The incidence of simian crease was $14.6 \%$.This incidence was highly significant $(p<0001)$ in Lama population $(71.2 \%)$. In these seven ethnic groups axial triradius angle was compared between those who had simian crease and with those who did not have simian crease. Comparisons were made statistically and found to be significant.

\section{Conclusion}

Incidence of simian crease in Nepalese children was $14.6 \%$ and was observed only in certain ethnic groups. It was significantly high in the Lama population $(71.2 \% 0$.

\section{Key Words}

Simian crease, single palmer crease, incidence, Down syndrome, ATD angle 


\section{INTRODUCTION}

Asimiancrease is a singleline that runsacross the palm ofthehand.Peoplenormallyhavethreecreasesintheir palms.Asingletransversepalmarcreaseformedbyfusion of the proximal and distal palmar creases, a common but not pathognomonic feature of Down syndrome; also found in $1 \%$ of thenormal population. ${ }^{1}$ The simian creaseisthemostmedicallyresearchedmarkerfoundon thehandsbecauseitis themostnoticeable.Averyhigh percentageofDownsyndromechildrenhavethismarker, however,thisdoesn'tdoesnotmeaneveryonewiththis markerhasDown. Manyexceptionallyintelligent,highly evolved people have this marker. ${ }^{2,3}$ Extensiveworkhas beencarriedoutonseveralpopulations,racial,andethnic groupsbuttherehasbeennospecificdocumentedstudy for the Nepalese population so far.Theobjective of this studywastodeterminetheprevalenceofsimiancrease inapparentlynormalindividualoftheNepaleseethnicity residing in Pokhara.

\section{METHODS}

A total of 2,067 children ( 1,084 male and 983 female) from five years to 18 years was screened randomly in theoutpatient department(OPD) ofManipalTeaching Hospital, Pokhara over a period of 7 months ( $1^{\text {st }}$ June 2007-31 ${ }^{\text {st }}$ December2007).Fourchildrenwereexcluded fromthestudyastheywerecasesofDownsyndrome.The remaining2,063(1,082 malesand 981 females) subjects were the focus of the study. They were classified into differentethnicgroups.Informedconsentwasobtained from parents and subjects. Each hand was inspected carefullyforsimiancreaseandaxialtriradiusangle(ATD) angle.Observationswerecategorisedintogenderand right/left palm(s). All children with simian crease were asked to wash their hands thoroughly and dry them completely.Thepatientwasthenasked toputthe palm side of his hand on theinked stamp padand then asked toplanthispalmonacleanwhitenon-glazedpapersheet. Theresultantpattern'a'-'d'\&'t'triradiuswas marked with the help of a magnifying glass. With the help of a scale andaprotractor, the'ATD',anglewasmeasured.Datawas analysedusingZ-testfortestingtwosampleproportions used tofind outthestatisticalsignificancebetweenthe Lamacasteandotherethnicgroups-Brahamin, Gurung, Tamang,Newar,Chettri,andtheDalitsseparately.T-test wasused tofindoutthestatisticalsignificancebetween ATD angles with simian crease and ATD angles with no simian crease. In all test $p$ value $<0.001$ was considered statistically significant.

\section{RESULTS}

Outof 2,063 children 1,082 weremalesand 981 females. Whenclassifiedintodifferentethnicgroups,therewere 14 groups(Table1)withBahamanrankingthehighest.There were seven ethnic groups namely Brahaman, Gurung, Tamang, Lama, Newar,Chettriand Dalit having simian crease(Table2).Incidenceofsimiancreasewasnoted to be 14.6\%(302outof2063)withconfidenceinterval95\% $(13.2 \%, 16.3 \%)$ out of which $54 \%$ were male and $46 \%$ female (fig.1). Highest number of single palmar crease was observed in the Lama population. Out of 59Lamas 42(71.2\%)hadsinglepalmercrease. Thisincidencewas significantly high with $\mathrm{p}<0.002$ when compared with other ethnic groups who had simian crease (table 3 ). Observation wascomparedin both palms (rightto left) in both sexes(Table4).Itwasobserved that in males the simiancreasewasmoreon therighthand (76\%) than in left hand (24\%) while in females it was more on the left hand ( $71 \%)$ than in right hand (29\%).The ATD angle wascomparedforethnicgroupswhohadsimiancreases with the same ethnic groups who did not have simian crease(Table 5). Ethnic groups Brahamin, Gurung, Lama,Newar,Chettri,Dalithaveastatisticallysignificant difference in ATD angles with simian crease and ATD angleswithnosimiancrease.Figure2depictstheformat usedtotaketheimprintforsimiancreaseandFig 3 shows the photo and imprint of simian crease.

Table 1. Sex distribution in different ethnic groups (From $\mathrm{N}=2063$ (total), $\mathrm{n}=302$

\begin{tabular}{llll} 
N=2063 & Male & Female & Total \\
\hline Brahaman & $240(48.0 \%)$ & $260(52.0 \%)$ & 500 \\
Gurung & $109(54.5 \%)$ & $91(45.5 \%)$ & 200 \\
Tamang & $24(58.5 \%)$ & $17(41.5 \%)$ & 41 \\
Magar & $29(58.0 \%)$ & $21(42.0 \%)$ & 50 \\
Lama & $32(54.2 \%)$ & $27(45.8 \%)$ & 59 \\
Newar & $167(55.7 \%)$ & $133(44.3 \%)$ & 300 \\
Chettri & $187(53.4 \%)$ & $163(46.6 \%)$ & 350 \\
Dalit & $139(50.5 \%)$ & $136(49.5 \%)$ & 275 \\
Rai & $12(75.0 \%)$ & $4(25.0 \%)$ & 16 \\
Muslim & $14(63.6 \%)$ & $8(36.4 \%)$ & 22 \\
Thakali & $7(58.3 \%)$ & $5(41.7 \%)$ & 12 \\
Tibeten & $14(50.0 \%)$ & $14(50.0 \%)$ & 28 \\
Pun & $48(48.0 \%)$ & $52(52.0 \%)$ & 100 \\
Madise & $44(55.0 \%)$ & $36(45.0 \%)$ & 80 \\
Other minority & $16(53.3 \%)$ & $14(46.7 \%)$ & 30 \\
groups & 1082 & 981 & 2063 \\
Total & & & \\
& & & 20 \\
\hline
\end{tabular}


Thincidenceofsimiancreaseis 14.6\%(302outof2063) withconfidenceinterval95\%(13.2\%,16.3\%)andmale 15.1\% ( 163 out of 1083 ) with confidence interval $95 \%(13.0 \%, 17.4 \%)$ and female $14.2 \%$ (139 out of 981 ) with confidence interval 95\% (12.1\% 16.5\%).

Table 2. Ethnic groups having Simian Crease

\begin{tabular}{lcccc|}
\hline N=302 & Male & Female & Total & percentage \\
\hline Brahaman $(n=500)$ & $44(48.4 \%)$ & $47(51.6 \%)$ & 91 & $18.2 \%$ \\
Gurung $(n=200)$ & $14(42.4 \%)$ & $19(57.6 \%)$ & 33 & $16.5 \%$ \\
Tamang $(n=41)$ & $1(100.0 \%)$ & $0(0.0 \%)$ & 1 & $2.4 \%$ \\
Lama $(n=59)$ & $26(61.9 \%)$ & $16(38.1 \%)$ & 42 & $71.2 \%$ \\
Newar $(n=300)$ & $19(54.3 \%)$ & $16(45.7 \%)$ & 35 & $11.6 \%$ \\
Chettri $(n=350)$ & $38(61.3 \%)$ & $24(38.7 \%)$ & 62 & $17.7 \%$ \\
Dalit $(n=275)$ & $21(55.3 \%)$ & $17(44.7 \%)$ & 38 & $13.8 \%$ \\
Total & $163(54 \%)$ & $139(46 \%)$ & 302 &
\end{tabular}

Table3.Comparison betweenEthnicgroups

\begin{tabular}{ll} 
Ethnic groups & P value \\
\hline Lama $(71.2 \%)$ & $0.0002^{*}$ \\
\hline Brahaman (18.2\%) & $0.0002^{*}$ \\
Gurung (16.5\%) & $0.0002^{*}$ \\
Tamang (2.4\%) & $0.0002^{*}$ \\
Newar (11.6\%) & $0.0002^{*}$ \\
Chettri $(17.7 \%)$ & $0.0002^{*}$ \\
Dalit $(13.8 \%)$ & $0.0002^{*}$ \\
* P value $<0.001$ statistically significant
\end{tabular}

Table 4. Sex and hand (right Vs Left) distribution of Simian Crease in different Ethnic groups

\begin{tabular}{|c|c|c|c|c|c|c|c|c|c|}
\hline \multirow{4}{*}{ Ethnic group } & \multicolumn{9}{|c|}{ Simian crease } \\
\hline & \multicolumn{4}{|c|}{ (Male $n=163)$ ) } & \multicolumn{4}{|c|}{ Female (139) } & \multirow{3}{*}{ Total } \\
\hline & \multicolumn{2}{|c|}{ Right } & \multicolumn{2}{|c|}{ left } & \multicolumn{2}{|c|}{ Right } & \multicolumn{2}{|c|}{ Left } & \\
\hline & No. & $\%$ & No. & $\%$ & No. & $\%$ & No. & $\%$ & \\
\hline Brahaman (500) & 30 & $18.4 \%$ & 14 & 8.5 & 7 & 5 & 40 & 28.7 & 91 \\
\hline Gurung (n=200) & 10 & 6.1 & 4 & 2.4 & 8 & 5.7 & 11 & 7.9 & 33 \\
\hline Tamang $(n=41)$ & 1 & 0.6 & 0 & 0 & 0 & 0 & 0 & 0 & 1 \\
\hline Lama (n=59) & 20 & 12.2 & 6 & 3.6 & 8 & 5.7 & 8 & 5.7 & 42 \\
\hline Newar $(n=300)$ & 15 & 9.2 & 4 & 2.4 & 6 & 4.3 & 10 & 7.2 & 35 \\
\hline Chettri ( $n=350)$ & 30 & 18.4 & 8 & 4.9 & 4 & 2.8 & 20 & 14.4 & 62 \\
\hline Dalit (n=275) & 18 & 11 & 3 & 1.8 & 7 & 5 & 10 & 7.2 & 38 \\
\hline Total & 124 & $76 \%$ & 39 & $24 \%$ & 40 & $29 \%$ & 99 & $71 \%$ & 302 \\
\hline
\end{tabular}

\section{DISCUSSION}

Asimiancreaseisasinglepalmarcreaseascomparedtotwo creasesinanormalpalm.Simiancreaseoccursinabout 1 outof30normalpeople,butisalsofrequentlyassociated withotherconditionssuchasDownsyndrome,Aarskog syndrome or fetalalcohol syndrome. ${ }^{4}$ The incidence of simiancreaseinnormal Nepalesechildrenwasstudied. The criteria to be met for normal children in this study wasachildwhodidnothaveanyfeaturesofchromosomal anomalies or other anomalies and was free from any chronicillnesses. Theincidenceofsimiancreaseinnormal Nepalese children was $14.6 \%$. About $10 \%$ of the Asian populationhasasimiancrease, andstudiesinthehands ofAfricanpygmieshaveshownpercentagescloseto $30 \%{ }^{3}$ In another study by Su C. Oyinbo \& H. Fawehinmi the incidencewas $4.14 \% .{ }^{5}$ Simiancreasehasbeenimplicated inmorethantwentyhumandiseases/geneticdisorders, ${ }^{6}$ includingDown'ssyndrome ${ }^{7}$ andintheaetiologyofseveral diseases such as leprosy ${ }^{8}$ and cancer, ${ }^{9}$ and rheumatoid arthritis. ${ }^{10}$ Unlikethisfindingallthechildreninthisstudy whowerefoundtohavesimiancreasewasnormalasallthe abovementionedillnesseswereexclusioncriteriaforthis study.InthisstudysevenethnicgroupsnamelyBrahman
(18.2\%), Gurung (16.5\%), Tamang (2.4\%), Lama (71.2\%), Newar(11.6\%),Chettri(17.7\%) and dalit 13.8\% )hadSimiancrease.Comparableliteratureforthiswasnot availablebutthefollowingpercentagesarepresentedas indicativeforvariousethnicpopulations:Pygmies:34.7 $\%$, Gypsies: $14.3 \%$, Chinese: $13.0 \%$, Koreans: $11.2 \%$, Kyushu Japanese: $9.2 \%$, Arabs \& Berbers: $7.9 \%$, Jews: $4.6 \%$, eastern Japanese: $4.0 \%$, Germans: $2.8 \%$, Ainu: $2.2 \%$, Dutch: $1.5 \%$, Eskimos: $1.3 \%$, Swiss: $1.2 \% .{ }^{11,3} \mathrm{It}$ wasobservedthat $54 \%$ malesand $46 \%$ femaleshadsimian creasewhichwas 3.98\%and $4.27 \%{ }^{12}$ inanotherstudyby Tsai FJ, Tsai CH, Peng CT et al .Yet in another study the prevalenceofsimiancreasewas $1.8 \%$ inmaleand $2.4 \%$ in femaleofthestudiedpopulation.Thegeneralpopulation prevalencewas $4.1 \% .{ }^{5}$ Inthisstudytheepisodeofsimian creaseinmaleswasmoreontherightpalm(76\%) versus leftpalm(24\%)whileinfemalesitwasmoreontheleftpalm (71\%) versusrightpalm(29\%).Unlikethisstudy in some otherstudysimiancreaseepisodedid notdiscriminate betweentherightand theleftpalm ${ }^{5,13}$ Proximal toeach fingeranapexatriradiusisformed bythedermalridgesby whichexactcentreofeachmountunderthefingercanbe 
Table 5. Comparision of ATD angle with and without Simian Crease

$\begin{array}{llll}\text { Ethnic groups } & \begin{array}{l}\text { ATD angles with Simian } \\ \text { Crease } \\ \text { Mean } \pm \text { SD }\end{array} & \begin{array}{l}\text { ATD angles with NO } \\ \text { Simian Crease } \\ \text { Mean } \pm \text { SD }\end{array} & \text { P value } \\ \text { Brahaman } & 36.16 \pm 3.6 & 41.77 \pm 5.5 & 0.00^{*} \\ \text { Gurung } & 35.82 \pm 3.7 & 41.76 \pm 5.1 & 0.00^{*} \\ \text { Lama } & 36.40 \pm 3.6 & 41.43 \pm 5.6 & 0.00^{*} \\ \text { Newar } & 35.46 \pm 3.3 & 42.37 \pm 6.0 & 0.00^{*} \\ \text { Chettri } & 35.29 \pm 3.2 & 41.81 \pm 5.3 & 0.00^{*} \\ \text { Dalit } & 36.24 \pm 3.2 & 41.61 \pm 5.7 & 0.00^{*}\end{array}$

* P value $<0.001$ statistically significant

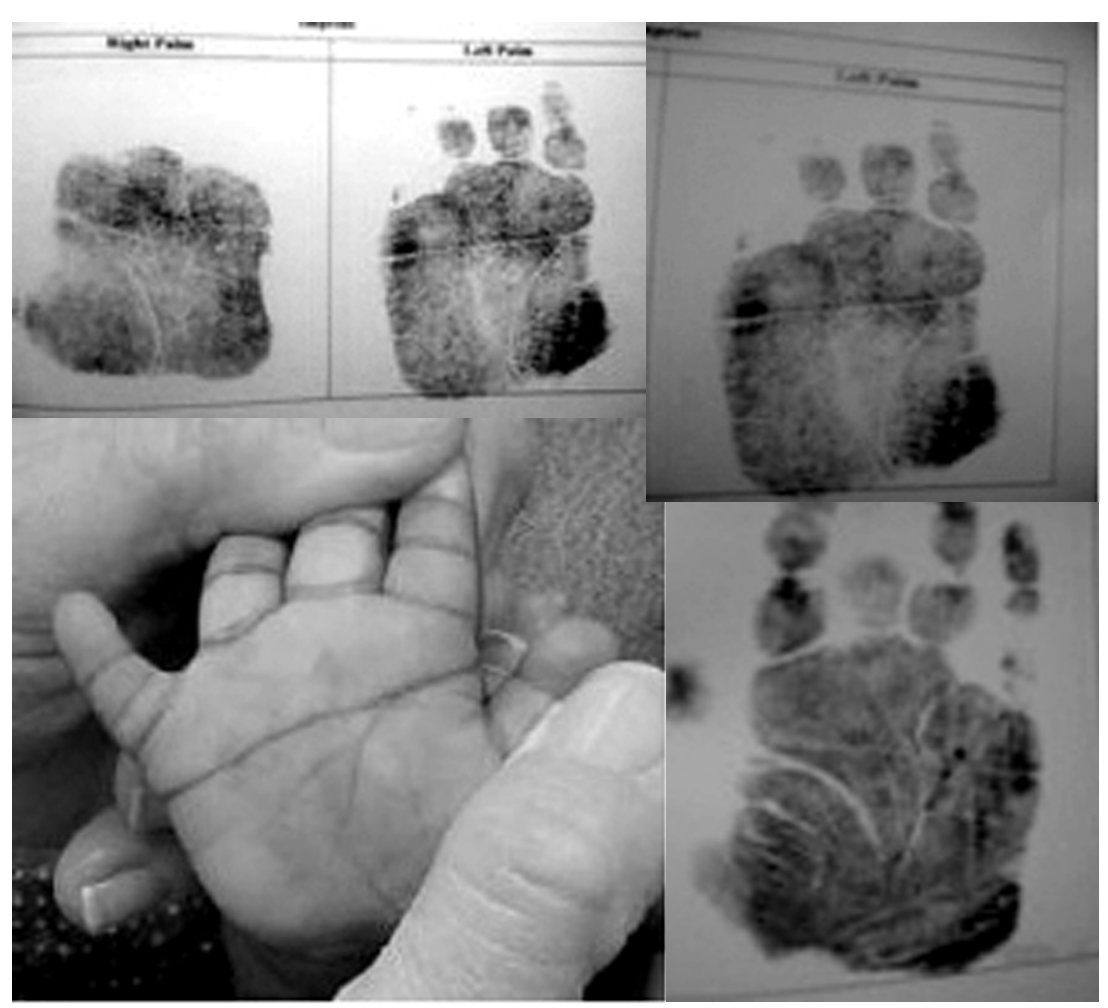

Figure 1. Simian Crease - Imprints and Image

located.Thesetriradiilocated proximaltothefingersare knownas'a,'b','c'and'd'triradiusstarting from thethumb tothelittlefingerrespectively.Thetriradiusontheproximal palminthecenterisknownas't'.Thelinejoining the 'a,', $d$ ' and't'triradiusformsangles'atd,'adt'and'dat.'.14Innormal being, the 'atd' angle ranges between $40^{\circ}-45^{\circ} .{ }^{14}$ In the presentstudytheATDanglewasobservedtobearound 41-42 ingroups with nosimiancreaseand was around 35-36 ingroupswith simiancrease.The ATDanglewas compared toethnicgroupswhohad simiancreasewith sameethnicgroupswhodidnothavesimiancrease.The 'ATD' angle of groups with no simian crease Brahaman (41.77 \pm 5.5$)$, Gurung (41.76 \pm 5.1$)$, Lama (41.43 \pm 5.6$)$, Newar (42.37 \pm 6.0$)$, Chettri (41.81 \pm 5.3), Dalit (41.61 $\pm 5.7)$ respectively was significantly $(p<0.0001)$ higher thanthatofgroupwithsimiancreaseBrahaman(36.16 \pm 3.6), Gurung (35.82 \pm 3.7$)$ Lama $(36.40 \pm 3.6)$,) Newar (35.46 \pm 3.3$)$. Chettri $(35.29 \pm 3.2)$ and Dalit (36.24 \pm 3.2) respectively.

\section{CONCLUSION}

Itcanbeconcluded thateveryonewithsmiancrease do notalsohaveDownsyndrome.14.6\%ofnormal Nepalese children have single palmer crease and is present only in certain ethnic groups with incidence being highly significant in the Lama population (71.2\%).Further screeningswithalargernumberofsubjectsmayprovidea better incidence.

\section{ACKNOWLEDGMENT}

Theauthorsaregratefultoallthechildrenand parentsfor theircooperationthroughoutthestudy, especiallyforcooperating to take imprint of simian crease. 


\section{REFERENCES}

1. MooreandDalley.Surfaceanatomyofthehand:Clinically Oriented Anatomy 5th edition, Lippincott Williams and Wilkins:Baltimore;2006.p.845-7.

2. HernandezM.PalmarcreasesinSpaniards.AnthropolAnz 1985;43:187-90.

3. Lestrange, M.T. de. The transverse crease in Europe: Index and comparative study of different samples cited intheliterature.American JournalofPhys.Anthropology 1960;35:2005-232.

4. KumbnaniHK.Dermatoglyphics:AReviewAnthropologist 2007 Special Volume No. 3:285-295

5. OyinboC,FawehinmiH.PrevalenceofsimianandSydney creases in the ljaws of South-South Nigeria. The Internet JournalofBiologicalAnthropology.2009Volume3Number 1:34-39

6. Loesch D. Dermatoglyphic distances and position of 21 trisomy mosaics. J Ment Defic Res 1979;23:253-63.

7. RignellA.Simiancreaseincidenceandthecorrelation with thenarandhypothenarpatterntypesinSwedish patients withtrisomy 21 (Down'ssyndrome).AmJPhys Anthropol 1987;72:277-86.
8. Gupta CM, Tutakne MA. An evaluation of palmar flexion creasesanddermatoglyphicsinleprosy.IndianJLepr 1986; 58:263-75.

9. JohannesHM,OzgenHM,KosterJ.PrevalenceandPatterns ofMorphologicalAbnormalitiesinPatientswithChildhood Cancer JAMA 2008;299:61-9.

10. Ravindranath R, Shubha R, Nagesh HV, et al. Dermatoglyphicsinrheumatoidarthritis. IndianJMedSci 2003;57:437-41.

11. Nazarabadi M, Raoofian R, Abutorabi1 R, Hosseini HB. Dermatoglyphic Assessment in Down and Klinefelter Syndromes. Iran J Med Sci 2007;32:105-9.

12. Tsai FJ, Tsai CH, Peng CT, Wu JY, Lien $\mathrm{CH}$, Wang TR Differentrace, differentface:minoranomaliesinChinese newborn infants. Acta Paediatr 1999;88:323-6.

13. Kamali MS. Simian crease polymorphism among fifteen Iranianendogamousgroups.AnthropolAnz1985;43:217-25.

14. Maskey D, Bhattacharya S, DhungelS, Jha CB, ShresthaS, GhimireSR,etal.Utilityofphenotypicdermalindicesinthe detection of DownsyndromepatientsNMCJ 2007:9:1-5. 